Gynecologic and

Obstetric Investigation
Gynecol Obstet Invest 2019;84:616-622

DOI: $10.1159 / 000501254$
Received: May 8, 2018

Accepted after revision: May 27, 2019

Published online: July 29, 2019

\title{
Early-Pregnancy Circulating Antioxidant Capacity and Hemodynamic Adaptation in Recurrent Placental Syndrome: An Exploratory Study
}

\author{
Carmen A.H. Severens-Rijvers ${ }^{a}$ Salwan Al-Nasiry ${ }^{b}$ Annemiek Vincken ${ }^{c}$ \\ Guido Haenen $^{d}$ Bjorn Winkens ${ }^{e}$ Chahinda Ghossein-Doha ${ }^{b}$ \\ Marc A.E. Spaanderman ${ }^{b}$ Louis L.H. Peeters ${ }^{f}$ \\ ${ }^{a}$ Department of Pathology, Maastricht University Medical Centre, Maastricht, The Netherlands; ${ }^{b}$ Department of \\ Obstetrics and Gynecology, Maastricht University Medical Centre, Maastricht, The Netherlands; ' ${ }^{\mathrm{D}}$ Department of Family \\ Medicine, Maastricht University Medical Centre, Maastricht, The Netherlands; ${ }^{\text {Department }}$ of Pharmacology and

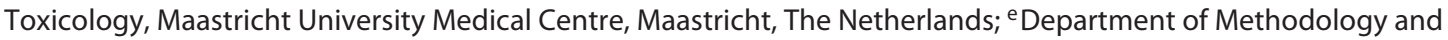 \\ Statistics, Maastricht University Medical Centre, CAPHRI Research School, Maastricht, The Netherlands; ${ }^{\mathrm{f}}$ Department of \\ Obstetrics, UMC Utrecht, Division "Vrouw and Baby", Utrecht, The Netherlands
}

\section{Keywords}

Preeclampsia · Hypertension in pregnancy · Pregnancy · Antioxidants · Trolox equivalent antioxidant capacity · Uric acid $\cdot$ Longitudinal

\footnotetext{
Abstract

Background/Aims: Placental syndromes (PS) refer to pregnancy complications that include gestational hypertension, (pre)eclampsia, HELLP syndrome, and/or placental insufficiency-induced fetal growth restriction. These disorders are characterized by increased oxidative stress. This study aims to test the hypothesis that the abnormal hemodynamic adaptation to pregnancy, typical for early PS pregnancy, is accompanied by abnormal maternal levels of antioxidants relative to those in normal pregnancy. Methods: Before, and at 12,16 , and 20 weeks pregnancy, we measured trolox equivalent antioxidant capacity (TEAC), uric acid (UA), and TEACC (TEAC corrected for UA) in maternal serum of former PS patients, who either developed recurrent PS (rPS; $n=16)$ or had
}

a normal next pregnancy (non-rPS; $n=23$ ). Concomitantly, we also measured various hemodynamic variables. Results: rPS differed from non-rPS by higher TEACC levels before pregnancy (178 vs. $152 \mu \mathrm{M} ; p=0.02$ ) and at 20 weeks pregnancy (180 vs. $160 \mu \mathrm{M} ; p=0.04$ ). Only non-rPS responded to pregnancy by significant rises in hemodynamic measures. Conclusion: These data indicate that rPS pregnancies are preceded by an increase in antioxidant capacity, presumably induced by subclinical vascular injury and low-grade chronic inflammation.

(c) 2019 The Author(s) Published by S. Karger AG, Basel

\section{Introduction}

Preeclampsia $(\mathrm{PE})$ is a common placental syndrome (PS) and contributes importantly to maternal and perinatal morbidity and mortality worldwide. Its overall prevalence is about $4.6 \%$ [1] with a recurrence rate of about

\begin{tabular}{ll}
\hline KARGER & $\begin{array}{l}\text { (c) } 2019 \text { The Author(s) } \\
\text { Published by S. Karger AG, Basel }\end{array}$ \\
E-Mail karger@karger.com & $\begin{array}{l}\text { This article is licensed under the Creative Commons Attribution- } \\
\text { NonCommercial-NoDerivatives 4.0 International License (CC BY- }\end{array}$ \\
www.karger.com/goi & $\begin{array}{l}\text { NC-ND) (http://www.karger.com/Services/OpenAccessLicense). } \\
\text { Usage and distribution for commercial purposes as well as any dis- } \\
\text { tribution of modified material requires written permission. }\end{array}$
\end{tabular}

Carmen A.H. Severens-Rijvers

Department of Pathology, Maastricht University Medical Centre CAPHRI Research School, Division "Vrouw and Baby"

NL-6202 AZ Maastricht (The Netherlands)

E-Mail carmen.rijvers@mumc.nl 
25\% [2]. Other PSs are gestational hypertension, HELLP syndrome, eclampsia, and fetal growth restriction caused by placental insufficiency. Although the clinical signs of PS usually only develop in the second half of pregnancy, PS is often preceded by impaired placental development in early pregnancy [3].

During early placentation, trophoblast cells (TBs) migrate upstream into the lumen of the spiral arteries, not only increasing their functional capacity but also forming plugs that impede maternal blood to reach the intervillous spaces. The latter not only secures embryonic development in a low oxygen environment. It also provides protection of proliferating and differentiating TBs against damage by reactive oxygen species (ROS) [4]. Defective spiral artery remodeling increases the risk of premature dislocation of TB plugs, and with it, premature rise in local tissue $\mathrm{pO}_{2}$ [5]. Therefore, the length of the period of hampered spiral artery blood flow and associated preservation of the low-oxygen milieu at the fetal-maternal interface contributes importantly to the embryo's defense against ROS, more so as fetal antioxidant defenses are relatively weak in early gestation [6]. These inferences support the view that circulating maternal antioxidant levels in early pregnancy are likely to provide an important back-up system in the defense of the developing concept against ROS. However, it is unknown whether this premature unplugging is the only cause of increased oxidative stress and development of clinical disease or whether other factors play a role as well. Antioxidant capacity also provides an indirect estimate for the amount of oxidative stress [7].

Antioxidants have been shown to be decreased in both plasma [8-10] and placentas [9] of women with PE. Unfortunately, in the preclinical phase of $\mathrm{PE}$, only a limited number of antioxidant markers were reported instead of total antioxidant capacity [9]. Therefore, we evaluated total antioxidant capacity by measuring trolox equivalent antioxidant capacity (TEAC), in serum of former PS patients before pregnancy, and again at 12,16, and 20 weeks of pregnancy.

\section{Methods}

We performed a retrospective longitudinal study at the Maastricht University Medical Center in Maastricht, the Netherlands. Although serum samples were collected prospectively, the samples were analyzed retrospectively. From 2002 to 2010, women who had a severe PS in the preceding pregnancy underwent serial monitoring in a specialized obstetrical unit before and during the first half of their next pregnancy as part of their high-risk obstetrical care. Exclusion criteria for this study were multiple pregnancies and presence of hypertension at the time of initial screening, since preexistent hypertension is not only treated in most cases but also associated with subnormal antioxidant levels [11]. The study protocol was approved by the institutional Medical Ethics reviewing Committee (MEC 10-4-049). All women gave informed consent and participated consecutively.

During the study period, we followed women prospectively with serial examination of various hemodynamic parameters and serum markers of the metabolic and the oxidative status. Post hoc, we subdivided these women into subgroups based on whether they did recurrent PS (rPS) or did not develop non-rPS later on in that pregnancy.

We defined PE and gestational hypertension, as described in detail previously [12]. A newborn was considered small-for-gestational-age, when birthweight was below the 10th centile, based on the most recent Dutch birth weight reference curves [13]. PS was considered severe, when its clinical onset was early ( $<34$ weeks) and/or the infant's birthweight was low ( $<2.3$ rd centile) due to placental dysfunction as diagnosed on the basis of abnormal fetal biometry (asymmetric growth) and/or abnormal Doppler tracings of the uterine arteries (resistance or pulsatility index $>90$ th centile).

After an overnight fast, we collected a venous blood sample, which was immediately centrifuged and the serum stored at $-30^{\circ} \mathrm{C}$ for later analysis. A technician blinded for pregnancy outcome measured all serum TEAC and uric acid (UA) levels after completion of data collection. We obtained ABTS (2,2'-azinobis [3-ethylbenzthiazoline-6-sulfonic acid]) and UA from Sigma Chemical Co. (St. Louis, MO, USA), and 2,2'-azino-bis(2-amidinopropane) and dihydrochloride from Brunschwig Chemicals (Amsterdam, The Netherlands). Finally, Merck Biochemica (Darmstadt, Germany) provided $\mathrm{NaH}_{2} \mathrm{PO}_{4} \cdot \mathrm{H}_{2} \mathrm{O}, \mathrm{Na}_{2} \mathrm{HPO}_{4} \cdot 2 \mathrm{H}_{2} \mathrm{O}$, and trichloroacetic acid, and $\mathrm{NaOH}$, which were of analytical grade purity. For measuring total serum antioxidant status, we used the TEAC assay. This assay determines the capacity of all antioxidants present in blood serum to scavenge ABTS ${ }^{\bullet}$. Serum deproteination was carried out by adding an equal volume of a $10 \%(\mathrm{w} / \mathrm{v})$ trichloroacetic acid solution. Serum samples were placed on ice for $5 \mathrm{~min}$ to complete deproteination, followed by centrifugation at $14,000 \mathrm{~g}$ at $4{ }^{\circ} \mathrm{C}$ for $5 \mathrm{~min}$. In the TEAC decolorization assay, $\mathrm{ABTS}^{\bullet+} \mathrm{ABTS}^{\bullet+}$ is generated chemically. ABTS ${ }^{*+}$ was produced by incubating a solution of $0.23 \mathrm{~mm}$ ABTS and $2.3 \mathrm{~mm}$ 2,2'-azino-bis(2-amidinopropane) dihydrochloride in $100 \mathrm{~mm}$ sodium phosphate buffer, $\mathrm{pH} 7.4$ at $70{ }^{\circ} \mathrm{C}$ until the absorption of the solution reached $0.70 \pm 0.02$ at $734 \mathrm{~nm}$. The antioxidants in the serum react with $\mathrm{ABTS}^{\circ+}$ which leads to decolorization of the initially blue/green sample. The fall in the $\mathrm{ABTS}^{\circ+}$ concentration in a given time period, due to the reaction of $\mathrm{ABTS}^{\bullet+}$ with the antioxidants present in the sample, is used to quantify the antioxidant capacity of the sample. So, the degree of decolorization reflects the amount of $\mathrm{ABTS}^{\cdot+}$ that has been scavenged and can be determined spectrophotometrically. The resulting value was compared with the TEAC value for the synthetic antioxidant Trolox (6-hydroxy-2,5,7,8-tetramethylchroman 2-carbonic acid). The TEAC value of a serum sample is expressed in $\mu \mathrm{M}$ and gives the level of a trolox solution, which corresponds to the antioxidant capacity in the serum sample.

Given that UA is a major determinant of TEAC [14] and also strongly associated with the development of PE [15], we also measured UA concentrations and calculated antioxidant capacity in serum attributable to other antioxidants than UA (TEACC). For 
Table 1. Baseline demographic characteristics and outcome of current pregnancy in both study groups

\begin{tabular}{|c|c|c|c|}
\hline & Non-rPS $(n=18)$ & $\operatorname{rPS}(n=21)$ & $p$ value \\
\hline Age, years & $33(30-36)$ & $32(29-36)$ & 0.57 \\
\hline BMI (at 12 weeks GA) & $27(23-31)$ & $27(23-28)$ & 0.47 \\
\hline $\mathrm{GH} / \mathrm{PE}, n(\%)$ & $15(83)$ & $20(95)$ & 0.22 \\
\hline SGA/stillbirth, $n(\%)$ & $3(17)$ & $1(5)$ & 0.22 \\
\hline Birth weight, $g$ & $1,630(974-2,509)$ & $1,283(670-1,841)$ & 0.22 \\
\hline \multicolumn{4}{|l|}{ Current pregnancy } \\
\hline $\mathrm{GH}, n(\%)$ & - & $10(48)$ & \\
\hline GA at onset, weeks & & $34.1(27-38)$ & \\
\hline $\mathrm{PE}, n(\%)$ & - & $6(29)$ & \\
\hline GA at onset, weeks & & $36.0(33-37)$ & \\
\hline SGA, $n(\%)$ & - & $8(38)$ & \\
\hline
\end{tabular}

Data are presented as median (IQR), unless noted otherwise.

BMI, body mass index; GA, gestational age; GH, gestational hypertension; PE, preeclampsia; SGA, small-for-gestational age; NonrPS, no recurrent placental syndrome; rPS, recurrent placental syndrome; IQR, interquartile range.

measuring UA in supernatant after denaturization, reversed-phase high-performance liquid chromatography was used. TEACC was calculated by subtracting UA from TEAC to provide an indication of the change in other important contributors to overall antioxidant capacity.

Since oxidative stress not only impairs hemodynamic function [16] but also associates with increased vascular injury- and inflammatory markers [17], we measured these components as well. PV was estimated as detailed previously [18]. Markers of vascular injury and inflammation (serum fibronectin and CRP, mg.L ${ }^{-1}$ ), parameters of maternal hemodynamic adaptations (mean arterial pressure $[\mathrm{mm} \mathrm{Hg}]$, heart rate $[\mathrm{bpm}]$, cardiac output $\left[\right.$ L.min $\left.{ }^{-1}\right]$, stroke volume $[\mathrm{mL}]$, creatinine clearance $\left[\mathrm{mL} \cdot \mathrm{min}^{-1}\right]$ ) [19], as well as uterine artery Doppler indices - as a measure of placental hemodynamic development - were performed as previously described [19-21].

We performed statistical analyses using IBM SPSS Statistics for Windows (version 23.0, Armonk, NY, USA). Data are presented as median with interquartile range for numerical variables and as number (\%) for categorical variables. Interquartile ranges are presented as 25 th and 75th percentiles. Intergroup differences were tested using Mann-Whitney $U$ test for numerical variables and Fisher's exact test for categorical variables. Spearman's rho correlations were used for associations between numerical variables. The differences in longitudinal trends in outcome parameters between groups (rPS vs. non-rPS) were assessed using linear mixed models with time (categorical: prepregnancy, 12, 16, and 20 weeks of pregnancy), group and group * time as fixed factors. Different covariance structures for repeated measures ([heterogeneous] first-order auto-regression, compound symmetry, diagonal, or independence) were considered, where the final structure was based on the smallest Akaike's information criterion. Missing outcome data are assumed to be missing at random, where the likelihoodbased approach (no multiple imputation) was used. Results from linear mixed models are presented as estimated means with standard error. A two-sided $p$ value $\leq 0.05$ was considered to be statistically significant.

\section{Results}

Serum samples were available from 48 women with a severe PS in their preceding pregnancy along with the intention to conceive again. We excluded 9 women because of preexistent hypertension, thus enrolling 39 women in our study. From these women, 16 developed rPS and 23 had a normal next pregnancy (non-rPS). Median time interval between pregnancies was in rPS 36 months (range 19-96 months) and in non-rPS 29 months (range 1792 months). The difference was not statistically significant $(p=0.866)$.

Table 1 lists baseline characteristics and pregnancy outcomes of both study groups. All women were Caucasian. There were no statistically significant differences between the 2 study groups, besides current pregnancy outcome in which rPS differed from that in non-rPS by an about 2 weeks shorter pregnancy duration $(p=0.003)$ and a $20 \%$ lower birthweight $(p=0.01)$.

Table 2 shows the levels of TEAC, UA, and TEACC at the 4 consecutive measurement points. There were no significant interaction effects of group with time. However, there was a higher TEACC level at 12 weeks 
Table 2. (Pre-)pregnant circulating levels of TEAC, UA, and TEACC

\begin{tabular}{|c|c|c|c|c|c|}
\hline & & Prepregnancy & 12 weeks GA & 16 weeks GA & 20 weeks GA \\
\hline & $\begin{array}{l}\text { Non-rPS } \\
\text { rPS }\end{array}$ & $\begin{array}{l}n=13 \\
n=9\end{array}$ & $\begin{array}{l}n=17 \\
n=20\end{array}$ & $\begin{array}{l}n=18 \\
n=20\end{array}$ & $\begin{array}{l}n=13 \\
n=16\end{array}$ \\
\hline \multirow{2}{*}{ TEAC, mM } & Non-rPS & 465 (19) & $389(15)^{* * *}$ & $394(16)^{* * *}$ & $398(23)^{* *}$ \\
\hline & rPS & $466(23)$ & $397(15)^{* * *}$ & $405(16)^{* *}$ & $437(24)$ \\
\hline \multirow[t]{2}{*}{$\mathrm{UA}, \mathrm{mM}$} & Non-rPS & $312(20)$ & $217(14)^{* * *}$ & $233(15)^{* * *}$ & $238(19)^{* * *}$ \\
\hline & rPS & $287(24)$ & $223(15)^{* *}$ & $230(15)^{*}$ & $258(20)$ \\
\hline \multirow[t]{2}{*}{ TEACC, mM } & Non-rPS & $152(7)$ & $171(6)^{* * *}$ & $161(6)$ & $160(7)$ \\
\hline & rPS & $178(8)^{\dagger}$ & $181(7)$ & $179(7)$ & $180(7)^{\dagger}$ \\
\hline
\end{tabular}

Data are presented as estimated means (SE), obtained from linear mixed model analysis.

† Significant difference $(p<0.05)$ compared to non-rPS.

* Significant within-group change $(p<0.05)$ compared to prepregnant value.

** Significant within-group change $(p<0.01)$ compared to prepregnant value.

*** Significant within-group change $(p \leq 0.001)$ compared to prepregnant value.

Non-rPS, no recurrent placental syndrome; rPS, recurrent placental syndrome; Pre, prepregnant; GA, gestational age; TEAC, trolox equivalent antioxidant capacity; UA, uric acid.

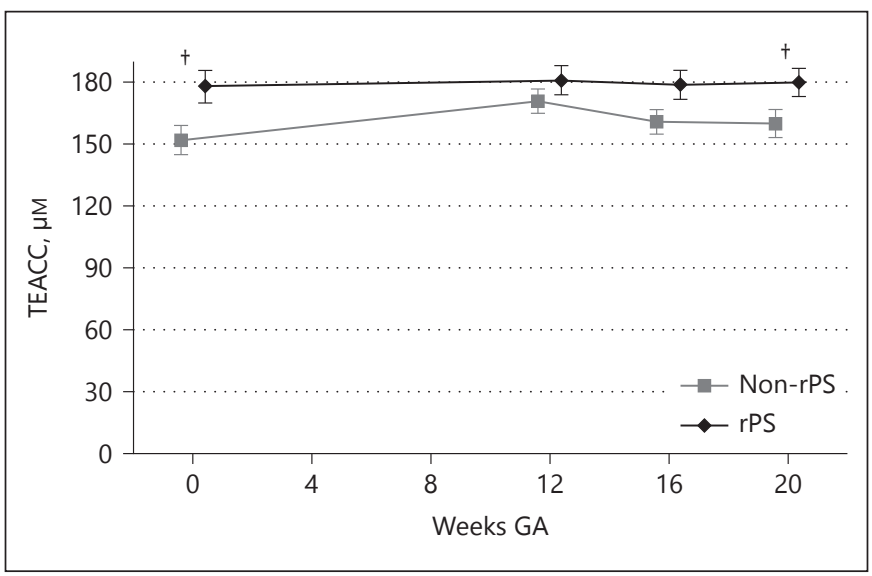

Fig. 1. Circulating TEACC concentrations in women with rPS and those without (non-rPS). Data are presented as estimated means $(\mathrm{SE})$, obtained from LMM analysis. ${ }^{\dagger}$ Significant difference $(p<$ $0.05)$ compared to non-rPS. GA, gestational age; rPS, recurrent placental syndromes; non-rPS, no recurrent placental syndrome; TEACC, trolox equivalent antioxidant capacity.

relative to prepregnancy, only in non-PS $(p=0.001)$. TEACC levels in rPS tended to be higher than in non-rPS, the difference only being statistically significant before pregnancy $(p=0.019)$ and at 20 weeks $(p=$ 0.041 ), as illustrated in Figure 1. Meanwhile, UA and TEAC levels did neither differ appreciably between the 2 groups at the 4 measurement points nor did these levels change appreciably during the measurement period, except for temporarily lower levels in both groups

Total Antioxidant Capacity in PS at 12 and 16 weeks, relative to prepregnancy (all $p<$ $0.05)$.

Table 3 shows maternal parameters related to inflammation and to the hemodynamic and placental adaptation to pregnancy in both study groups. Although the hemodynamic response to pregnancy was similar in both study groups, the magnitude of the systemic changes was larger in non-rPS. Fibronectin levels were higher in rPS at 16 weeks pregnancy than in non-rPS $(p=0.008)$, this difference being only borderline significant at 12 and 20 weeks ( $p=0.059$ and $p=0.056$, respectively). Prepregnant CRP seemed higher in rPS, but because there were only 2 women with data at that time-point in the rPS group, it was not possible to test for a between-group difference. However, in both groups we observed a rise in $\mathrm{CRP}$ in response to pregnancy (non-rPS $p \leq 0.001$ at 12 weeks, $\operatorname{rPS} p=0.042$ at 20 weeks of gestation), reaching a comparable plateau by 12 weeks. Interestingly, the highest uterine PI and RI levels at 12 weeks of pregnancy in the rPS group were observed in women who also had the highest TEACC levels $(r=0.736, p=0.028$ and $r=0.788$, $p=0.025$, respectively; online suppl. Table. 1 , see www. karger.com/doi/10.1159/000501254).

\section{Discussion}

In this study, we demonstrated that antioxidant capacity measured as TEACC levels was higher in rPS as compared to non-rPS in the prepregnant state and at 20 weeks 
Table 3. (Pre)pregnant maternal parameters for inflammation, hemodynamic, and placental adaptation to pregnancy in women with rPS and those without (non-rPS)

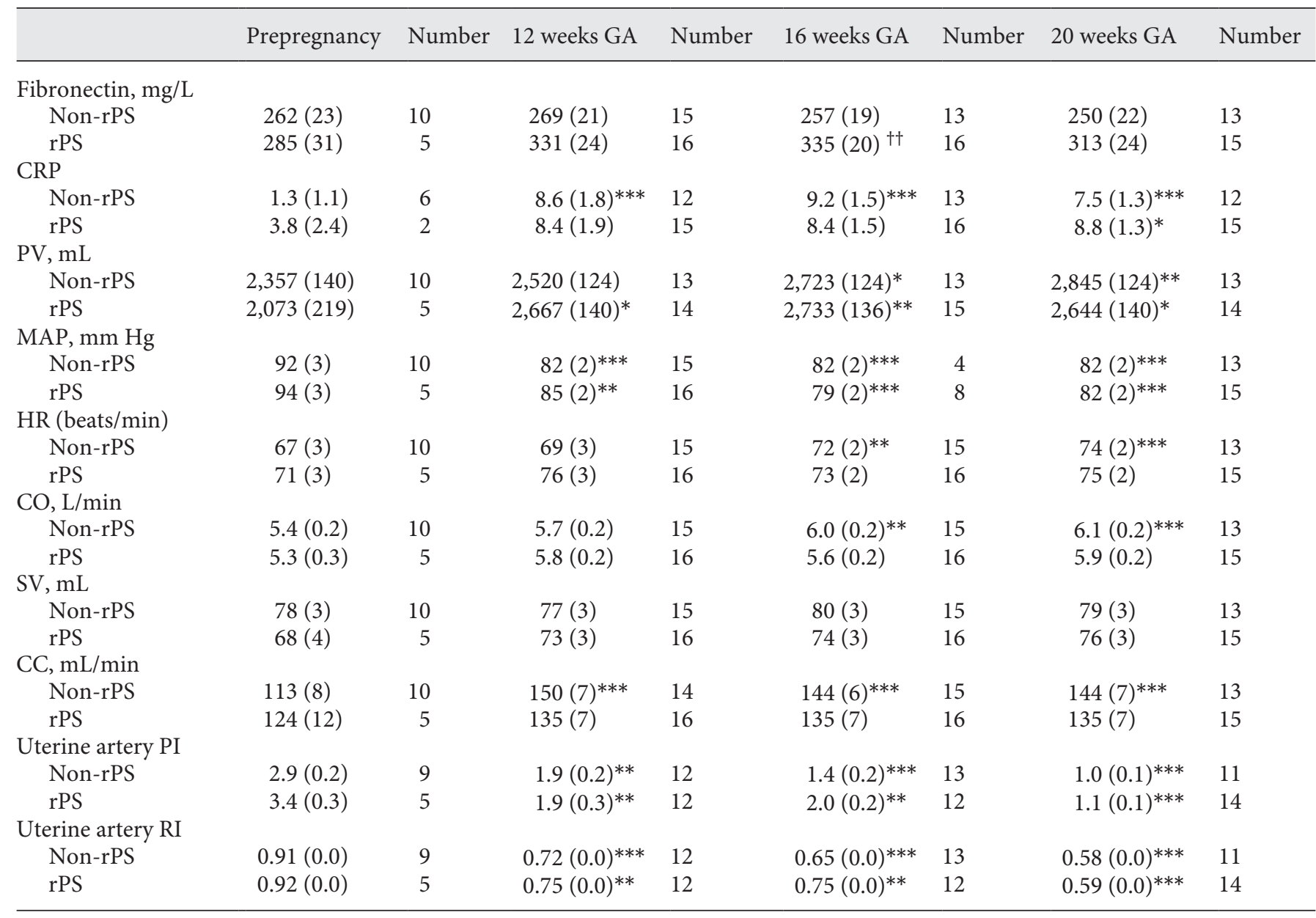

Data are presented as estimated means (SE) obtained from linear mixed model analysis.

* Significant within-group change $(p<0.05)$ compared to prpregnant value.

** Significant within-group change $(p \leq 0.01)$ compared to prepregnant value.

$* * *$ Significant within-group change $(p \leq 0.001)$ compared to prepregnant value.

t† Significant difference $(p<0.01)$ compared to Non-rPS.

CRP, C-reactive protein; PV, plasma volume; MAP, mean arterial pressure; PI, pulsatility index; RI, resistance index; PS, placental syndrome; non-rPS, no recurrent PS; rPS, recurrent PS; HR, heart rate; CO, cardiac output; SV, stroke volume; CC, creatinine clearance.

pregnancy. An increase was also seen in fibronectin levels, but these were only significantly higher in rPS at 16 weeks. Maternal hemodynamic response to pregnancy was similar in both groups, but the magnitude of the systemic changes was larger in non-rPS. CRP levels in pregnancy did not differ appreciably between both groups. We had insufficient pre-pregnant CRP data in this study to enable intergroup comparison.

Changes in circulating biomarkers for oxidative stress and antioxidant capacity as indicators of normal preg- nancy adaptation as well as predictors of the later development of PE have been debated for decades [22, 23]. We previously observed higher prepregnant CRP and fibronectin levels in a large fraction of former PS women, indicative of chronic low-grade inflammation and subclinical endothelial activatin [24]. Together with the observation in the present study of higher prepregnant TEACC levels, this suggests that the higher prepregnant TEACC levels in rPS patients in this study could be an adaptive response to the previously reported chronic changes. This 
adaptive response is supported by the strong positive correlation between TEACC and uterine artery Doppler PI/ RI values, since increased uterine PI and RI are consistent with defective spiral artery remodeling [25] and therefore with increased oxidative stress. Such a redox mediated, adaptive response is not unusual in nature [26].

Reports on the antioxidant levels in relation to PS are conflicting with some studies showing decreased [8-10] and others increased circulating antioxidant levels $[27,28]$ in PS pregnancies. Similarly, studies measuring antioxidant status in the preclinical phase of the disorder showed either decreased [29] or equal [30] levels. The conflicting reports are probably related to the use of different methods to assess the antioxidant status, for example, by determining distinct antioxidative enzymes or total antioxidant capacity or by correcting or not correcting the antioxidant status for UA levels, as the endocrine environment of pregnancy is known to influence UA levels [31].

Our findings do not support a solitary link between pregnancy-dependent changes in circulating antioxidant capacity and abnormal placental development in rPS. At any rate, it is conceivable that the preexistent subclinical vascular damage together with the chronic low-grade inflammation are responsible for both the trend to higher antioxidant capacity and the defective placental development, which precedes symptomatic rPS. Thus, rPS may continue to develop in spite of the already activated antioxidant system. Apparently, the activated antioxidant system in rPS pregnancies does not play a protective role, but is merely an epiphenomenon of some other latent cardiovascular or metabolic abnormality that interferes with early placental development. It is now widely accepted that PS is indeed related to (sub)clinical cardiovascular and metabolic changes. It is interesting to interpret these results in the context of larger randomized controlled trials which did not show any effect of antenatal antioxidant therapy with vitamin $\mathrm{E}$ and/or $\mathrm{C}$ in preventing $\mathrm{PE}[32,33]$.
Strengths of this study are the inclusion of longitudinal data on antioxidant capacity from preconception until mid-pregnancy and the simultaneous measurement of various circulatory parameters. This allowed us to study the association between oxidative stress and maternal hemodynamic adaptation in a group of women at high risk of developing PS. Yet, we are aware of the modest sample size that limits the power of this study. On the other hand, the demonstration of statistically significant differences in this exploratory study is encouraging and may enable sample size calculations for larger prospective studies. We are also aware of the consequence of studying this particular high-risk population, which is known to contain a relatively large fraction of women with some subclinical disorder that may influence the endpoints of this study.

In conclusion, this study showed that rPS pregnancies are preceded by an increase in antioxidant capacity, presumably induced by subclinical vascular injury and lowgrade chronic inflammation. More research is needed to determine how these phenomena are interconnected to improve the management strategy of high-risk pregnancies.

\section{Acknowledgments}

The authors want to acknowledge Timo Ekhart, Departments of Obstetrics and Gynecology, Maastricht University Medical Centre, Maastricht, The Netherlands, for the collection and entry of the data.

\section{Disclosure Statement}

The authors report no conflict of interest.

\section{Funding Sources}

There was no external funding of this study.

\section{References}

1 Abalos E, Cuesta C, Grosso AL, Chou D, Say L. Global and regional estimates of preeclampsia and eclampsia: a systematic review. Eur JObstet Gynecol Reprod Biol. 2013 Sep;170(1):1-7.

2 Sep SJ, Smits LJ, Prins MH, Spaanderman ME, Peeters LL. Simple prepregnant prediction rule for recurrent early-onset hypertensive disease in pregnancy. Reprod Sci. 2009 Jan;16(1):80-7.

3 Roberts JM, Hubel CA. The two stage model of preeclampsia: variations on the theme. Placenta. 2009;30(suppl A):S32-37.
4 Burton GJ, Jauniaux E. Placental oxidative stress: from miscarriage to preeclampsia. J Soc Gynecol Investig. 2004 Sep;11(6):34252.

5 Jauniaux E, Watson AL, Hempstock J, Bao YP, Skepper JN, Burton GJ. Onset of maternal arterial blood flow and placental oxidative stress. A possible factor in human early pregnancy failure. Am J Pathol. 2000 Dec;157(6): 2111-22.

6 Ornoy A. Embryonic oxidative stress as a mechanism of teratogenesis with special em- phasis on diabetic embryopathy. Reprod Toxicol. 2007 Jul;24(1):31-41.

7 Oxidative Stress. Diagnostic Methods and Applications in Medical Science. 1st ed. Springer; 2017.

8 D'souza JM, Harish S, Pai VR, Shriyan C. Increased Oxidatively Modified Forms of Albumin in Association with Decreased Total Antioxidant Activity in Different Types of Hypertensive Disorders of Pregnancy. Indian J Clin Biochem. 2017 Jun; 32(2):200-6. 
9 D’Souza V, Rani A, Patil V, Pisal H, Randhir $\mathrm{K}$, Mehendale S, et al. Increased oxidative stress from early pregnancy in women who develop preeclampsia. Clin Exp Hypertens. 2016;38(2):225-32.

10 Kirbas A, Daglar K, Gencosmanoglu G, Yilmaz Z, Timur H, Inal Z, et al. Total oxidative and anti-oxidative status, and ADAMTS-12 levels in placenta previa and early-onset severe preeclampsia. Pregnancy Hypertens. 2016 Oct; 6(4):295-9.

11 Pedro-Botet J, Covas MI, Martín S, RubiésPrat J. Decreased endogenous antioxidant enzymatic status in essential hypertension. J Hum Hypertens. 2000 Jun;14(6):343-5.

12 Report of the National High Blood Pressure Education Program Working Group on High Blood Pressure in Pregnancy. Am J Obstet Gynecol. 2000 Jul;183(1):S1-22.

13 Speer PD, Powers RW, Frank MP, Harger G, Markovic N, Roberts JM. Elevated asymmetric dimethylarginine concentrations precede clinical preeclampsia, but not pregnancies with small-for-gestational-age infants. Am J Obstet Gynecol. 2008;198:112 e111-117.

14 Miller NJ, Rice-Evans C, Davies MJ, Gopinathan V, Milner A. A novel method for measuring antioxidant capacity and its application to monitoring the antioxidant status in premature neonates. Clin Sci (Lond). 1993 Apr;84(4):407-12.

15 Bellomo G. Serum uric acid and pre-eclampsia: an update. Expert Rev Cardiovasc Ther. 2012 Jun;10(6):701-5.

16 Raaz U, Toh R, Maegdefessel L, Adam M, Nakagami F, Emrich FC, et al. Hemodynamic regulation of reactive oxygen species: implications for vascular diseases. Antioxid Redox Signal. 2014 Feb;20(6):914-28.

17 Severens-Rijvers CA, Al-Nasiry S, GhosseinDoha C, Marzano S, Ten Cate H, Winkens B, et al. Circulating Fibronectin and Plasminogen Activator Inhibitor-2 Levels as Possible
Predictors of Recurrent Placental Syndrome: An Exploratory Study. Gynecol Obstet Invest. 2017;82(4):355-60.

18 van Kreel BK, van Beek E, Spaanderman ME, Peeters LL. A new method for plasma volume measurements with unlabeled dextran-70 instead of 125I-labeled albumin as an indicator. Clin Chim Acta. 1998 Jul;275(1):71-80.

19 Ghossein-Doha C, Spaanderman ME, Al Doulah R, Van Kuijk SM, Peeters LL. Maternal cardiac adaptation to subsequent pregnancy in formerly pre-eclamptic women according to recurrence of pre-eclampsia. Ultrasound Obstet Gynecol. 2016;47:96-103.

20 Aardenburg R, Spaanderman ME, Ekhart TH, van Eijndhoven HW, van der Heijden OW, Peeters LL. Low plasma volume following pregnancy complicated by pre-eclampsia predisposes for hypertensive disease in a next pregnancy. BJOG. 2003 Nov; 110(11):1001-6.

21 Valensise H, Bezzeccheri V, Rizzo G, Tranquilli AL, Garzetti GG, Romanini C. Doppler velocimetry of the uterine artery as a screening test for gestational hypertension. Ultrasound Obstet Gynecol. 1993 Jan;3(1):18-22.

22 Basu J, Bendek B, Agamasu E, Salafia CM, Mishra A, Benfield N, et al. Placental Oxidative Status throughout Normal Gestation in Women with Uncomplicated Pregnancies. Obstet Gynecol Int. 2015;2015:276095.

23 Chappell LC, Seed PT, Briley A, Kelly FJ, Hunt BJ, Charnock-Jones DS, et al. A longitudinal study of biochemical variables in women at risk of preeclampsia. Am J Obstet Gynecol. 2002 Jul;187(1):127-36.

24 van Rijn BB, Veerbeek JH, Scholtens LC, Post Uiterweer ED, Koster MP, Peeters LL, et al. $\mathrm{C}$-reactive protein and fibrinogen levels as determinants of recurrent preeclampsia: a prospective cohort study. J Hypertens. $2014 \mathrm{Feb}$; 32(2):408-14.

25 Olofsson P, Laurini RN, Marsál K. A high uterine artery pulsatility index reflects a de- fective development of placental bed spiral arteries in pregnancies complicated by hypertension and fetal growth retardation. Eur J Obstet Gynecol Reprod Biol. 1993 May;49(3): 161-8.

26 Sthijns MM, Weseler AR, Bast A, Haenen GR. Time in Redox Adaptation Processes: From Evolution to Hormesis. Int J Mol Sci. 2016 Sep;17(10):17.

27 Keshavarz P, Nobakht M Gh BF, Mirhafez SR, Nematy M, Azimi-Nezhad M, Afin SA, et al. Alterations in Lipid Profile, Zinc and Copper Levels and Superoxide Dismutase Activities in Normal Pregnancy and Preeclampsia. Am J Med Sci. 2017 Jun;353(6):552-8.

28 Nikolic A, Cabarkapa V, Novakov Mikic A, Jakovljević A, Stosic Z. Ceruloplasmin and antioxidative enzymes in pre-eclampsia. J Matern Fetal Neonatal Med. 2016 Sep;29(18): 2987-93.

29 Cohen JM, Kramer MS, Platt RW, Basso O, Evans RW, Kahn SR. The association between maternal antioxidant levels in midpregnancy and preeclampsia. Am J Obstet Gynecol. 2015 Nov;213(5):695.e691-613.

30 Roes EM, Hendriks JC, Raijmakers MT, Steegers-Theunissen RP, Groenen P, Peters $\mathrm{WH}$, et al. A longitudinal study of antioxidant status during uncomplicated and hypertensive pregnancies. Acta Obstet Gynecol Scand. 2006;85(2):148-55.

31 Quiñones Galvan A, Natali A, Baldi S, Frascerra S, Sanna G, Ciociaro D, et al. Effect of insulin on uric acid excretion in humans. Am J Physiol. 1995 Jan;268(1 Pt 1):E1-5.

32 Rumbold A, Ota E, Hori H, Miyazaki C Crowther CA. Vitamin E supplementation in pregnancy. Cochrane Database Syst Rev. 2015 Sep;(9):CD004069.

33 Rumbold A, Ota E, Nagata C, Shahrook S, Crowther CA. Vitamin C supplementation in pregnancy. Cochrane Database Syst Rev. 2015 Sep;(9):CD004072. 